\title{
The expression of $\mathrm{NAD}(\mathrm{P}) \mathrm{H}$ :quinone oxidoreductase 1 is increased along with NF- $\mathrm{kB}$ p105/p50 in human cutaneous melanomas
}

\author{
YABIN CHENG ${ }^{1}$, JUN LI $^{1}$, MAGDALENA MARTINKA ${ }^{2}$ and GANG LI ${ }^{1}$ \\ Departments of ${ }^{1}$ Dermatology and Skin Science, and ${ }^{2}$ Pathology, Vancouver Coastal Health \\ Research Institute, University of British Columbia, Vancouver, B.C., Canada
}

Received October 5, 2009; Accepted November 30, 2009

DOI: $10.3892 /$ or_00000722

\begin{abstract}
NAD(P)H:quinone oxidoreductase 1 (NQO1) is a key enzyme involved in metabolism of quinones and may perform multiple functions within the cell. Recent studies demonstrated that NQO1 is overexpressed in many types of tumors, including the lung, ovary, adrenal gland, thyroid, liver, colon, breast, and pancreas. To investigate whether NQO1 plays a role in melanoma pathogenesis, we used tissue microarray technology and immunohistochemistry to examine NQO1 expression in 56 dysplastic nevi and 93 primary melanoma biopsies. Our data showed that NQO1 expression is significantly increased in primary melanomas compared with dysplastic nevi ( $\mathrm{P}=0.015, \chi^{2}$ test). Our results also revealed that the increase of NQO1 was not associated with patient age, tumor thickness, ulceration, tumor site, American Joint Committee on Cancer (AJCC) stage, and 5-year patient survival. Interestingly, we found that female patients had more NQO1 expression than male patients $\left(\mathrm{P}=0.022, \chi^{2}\right.$ test). Furthermore, NQO1 expression level was significantly higher in superficial spreading melanomas compared with other tumor subtypes ( $\mathrm{P}=0.020, \chi^{2}$ test). Moreover, we found that NQO1 expression is significantly correlated with the expression of NF- $\kappa \mathrm{B}$ subunit p50 ( $\mathrm{P}=0.032, \chi^{2}$ test $)$. Our findings suggest that NQO1 may play an important role in the initiation stage of melanoma development.
\end{abstract}

\section{Introduction}

Cutaneous malignant melanoma is a type of skin cancer originating from neoplastic proliferation of melanocytes in the epidermis. Due to its rapid lymphogenic and haematogenic

Correspondence to: Dr Gang Li, Jack Bell Research Centre, 2660 Oak Street, Vancouver, BC V6H 3Z6, Canada

E-mail: gangli@interchange.ubc.ca

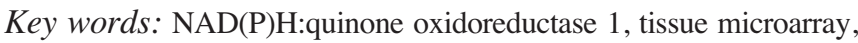
melanoma metastasis, melanoma is one of the most malignant human tumors (1). Melanoma comprises only $4-5 \%$ of all skin cancers, but it is responsible for $\sim 80 \%$ of deaths from skin cancer (2). Data from the National Cancer Institute (NCI) estimate that 62,480 new cases of melanoma of the skin are diagnosed in the United States this year, and $~ 8,420$ of patients will die from this disease (3). Although melanomas at early stages are curable with surgical excision, up to $20 \%$ of patients will develop metastatic tumors owing to its high capability of invasion and rapid metastasis to other organs $(4,5)$. Patients with metastatic melanoma have a poor prognosis, with a median survival of only $6-10$ months and $<5 \%$ of patients surviving more than 5 years (5-8). Therefore, understanding of the molecular events and mechanisms underlying the tumor initiation and progression, which could contribute to early detection, will be helpful for prevention and treatment of melanoma.

$\mathrm{NAD}(\mathrm{P}) \mathrm{H}$ :quinine oxidoreductase-1 (NQO1) is primarily a cytosolic enzyme that uses NADH or NADPH to directly reduce quinones to hydroquinones (9). The enzyme is generally considered as a detoxification enzyme because of its ability to detoxify reactive quinones and quinine-imines to less reactive and less toxic hydroquinones (10). This two-electron reductase also bypasses the highly reactive semi-quinone radical intermediate and prevents redox cycling (9). Through the detoxification of quinones, NQO1 prevents the formation of reactive oxygen species (ROS) and plays an important role in the cellular antioxidant system (11-14). However, the conclusions on the biological functions of NQO1 in cancer were contradictory. On one hand, NQO1 catalyzes the bioactivation of cytotoxic quinones, thereby, increasing their antitumor efficacy $(15,16)$. On the other hand, reductive activation of environmental carcinogens including dinitropyrenes and heterocyclic amines by NQO1 could contribute to carcinogenesis (17). Interestingly, NQO1 was found to be expressed at high levels in many solid tumors, such as adrenal gland, breast, colon, lung, ovary, pancreas and thyroid (18-22).

To date, studies on the regulation of NQO1 gene expression have shown a complex molecular pathway. The involvement of the nuclear factor kappaB (NF-кB) signalling pathway in the induction of NQO1 gene expression has been demonstrated in different animal models and cell lines $(23,24)$. Several studies showed that induction of $N Q O 1$ gene by 
hypoxia and mitomycin $\mathrm{C}$ in human colon adenocarcinoma HT29 and human hepatoma HepG2 cells is mediated through a mechanism involving the $\mathrm{NF}-\kappa \mathrm{B}$ signalling pathway $(23,24)$. NF-кB has been shown to play an important role in melanoma proliferation, resistance to apoptosis, invasion, and metastasis (25). Our previous studies has demonstrated that NF- $\mathrm{KB}$ p105/p50 nuclear staining is increased in melanoma compared with dysplastic nevi (26).

Despite the extensive interest in NQO1, there is little information regarding its expression in melanoma. The aim of the present study was to determine the expression of NQO1 in different stages of human melanocytic lesions and to further investigate the role of NQO1 activity in melanoma progression using tissue microarray (TMA) technology and immunohistochemistry. Our data showed that NQO1 expression is significantly increased in primary melanomas compared to dysplastic nevi. However, increased NQO1 expression is not correlated with 5-year patient survival. We also examined the correlation of NQO1 and NF-кB p105/p50 nuclear expression in 93 cases of primary melanoma. Our data showed that $\mathrm{NF}-\kappa \mathrm{B}$ nuclear staining is significantly increased with NQO1 expression, which indicates that NQO1 expression may be regulated through $\mathrm{NF}-\kappa \mathrm{B}$ signalling pathway.

\section{Materials and methods}

TMA construction. Formalin-fixed, paraffin-embedded tissue blocks containing 66 dysplastic nevi, 118 primary melanomas were used in our present study. All specimens were obtained from the 1990-1998 archives of the Department of Pathology at Vancouver General Hospital. The use of human skin tissues in this study was approved by the medical ethics committee of the University of British Columbia and was performed in accordance with the Declaration of Helsinki guidelines. For each case, the most representative tumor area was carefully selected and marked on the hematoxylin and eosin (H\&E)stained slide. The TMAs were assembled using a tissue-array instrument (Beecher Instruments, Silver Spring, MD). Taking into account the limitation of the representative areas of the tumor, we used duplicate $0.6 \mathrm{~mm}$-thick tissue cores from each donor block. Multiple $4 \mu \mathrm{m}$-sections were cut with a Leica microtome (Leica Microsystems Inc., Bannockburn, IL) and then transferred to adhesive-coated slides using routine histology procedures. One section from each TMA was routinely stained with $H \& E$. The remaining sections were stored at room temperature for immunohistochemical staining.

Immunohistochemistry of TMA. The TMA slides were heated at $55^{\circ} \mathrm{C}$ for $30 \mathrm{~min}$ to be dewaxed, and then washed with xylene 3 times, 5 min each. The tissues were then rehydrated in a series of 5-min washes in 100, 95, 80\% ethanol and distilled water. Antigen retrieval was performed by heating the samples at $95^{\circ} \mathrm{C}$ for $30 \mathrm{~min}$ in $10 \mathrm{mM}$ sodium citrate ( $\mathrm{pH} \mathrm{6.0)}$ ). Endogenous peroxidase activity was blocked with $3 \%$ hydrogen peroxide for $20 \mathrm{~min}$, and nonspecific binding was blocked by universal blocking serum (Dako Diagnostics, Mississauga, ON, Canada) for $30 \mathrm{~min}$. The primary monoclonal mouse anti-NQO1 antibody (Santa Cruz Biotechnology Inc., Santa Cruz, CA) was diluted 1:100 and incubated at $4^{\circ} \mathrm{C}$
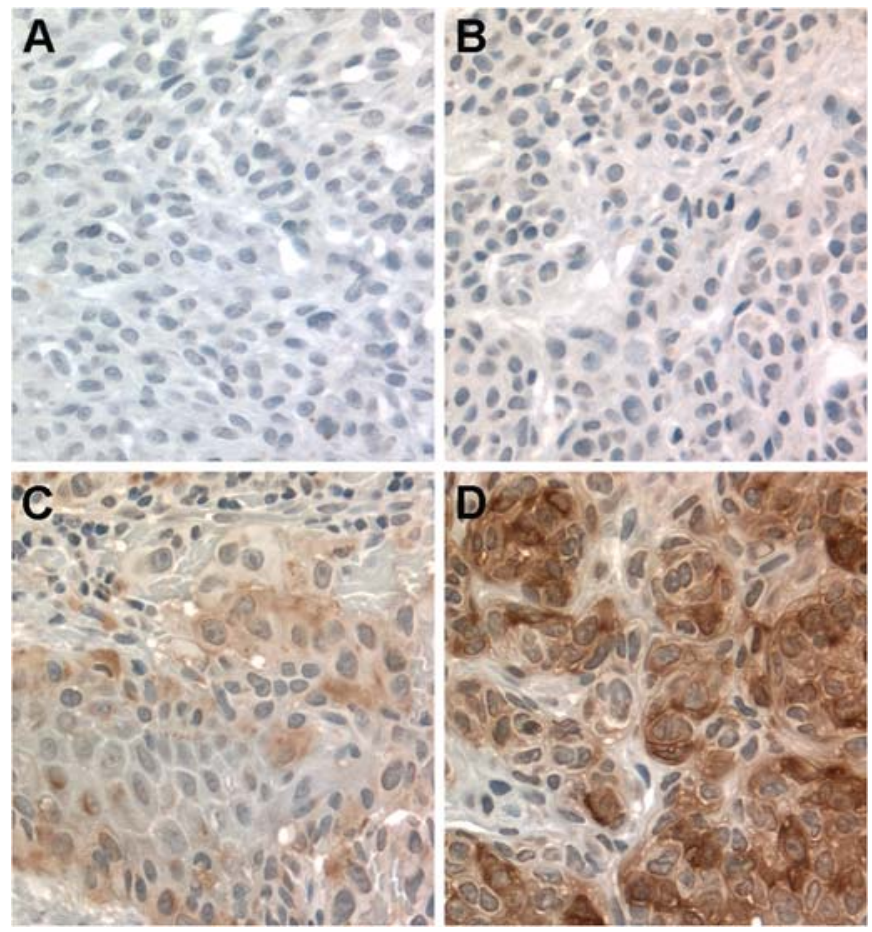

Figure 1. Representative images of NQO1 immunohistochemical staining in human melanocytic lesions. Dysplastic nevus with negative (A) or weak NQO1 expression (B). Primary melanoma with moderate (C) or strong NQO1 expression (D). Magnification, $x 400$.

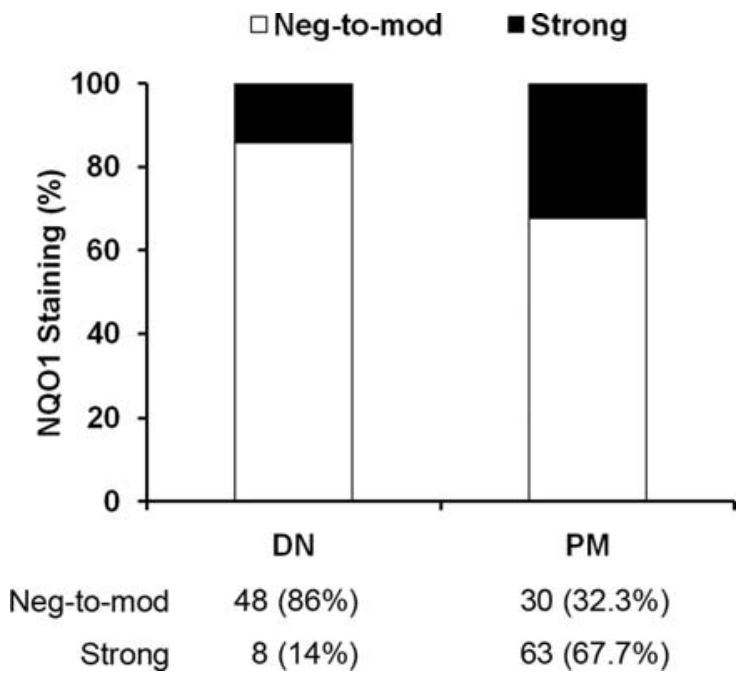

Figure 2. NQO1 expression in dysplastic nevi (DN) and primary melanoma (PM). Negative-to-moderate staining (0-9); strong staining (12). A significantly higher expression of NQO1 was found in primary melanoma when compared to dysplastic nevi $\left(\mathrm{P}=0.015, \chi^{2}\right.$ test $)$.

overnight. After 3 washes, 2 min each with PBS, the sections were incubated with biotin-labeled secondary antibody and streptavidin-peroxidase for $30 \mathrm{~min}$ each (Dako Diagnostics). The samples were then developed with 3,3'-diaminobenzidine substrate (Vector Laboratories, Burlington, Ontario, Canada) and counterstained with hematoxylin. Then the slides were dehydrated following a standard procedure and sealed with 

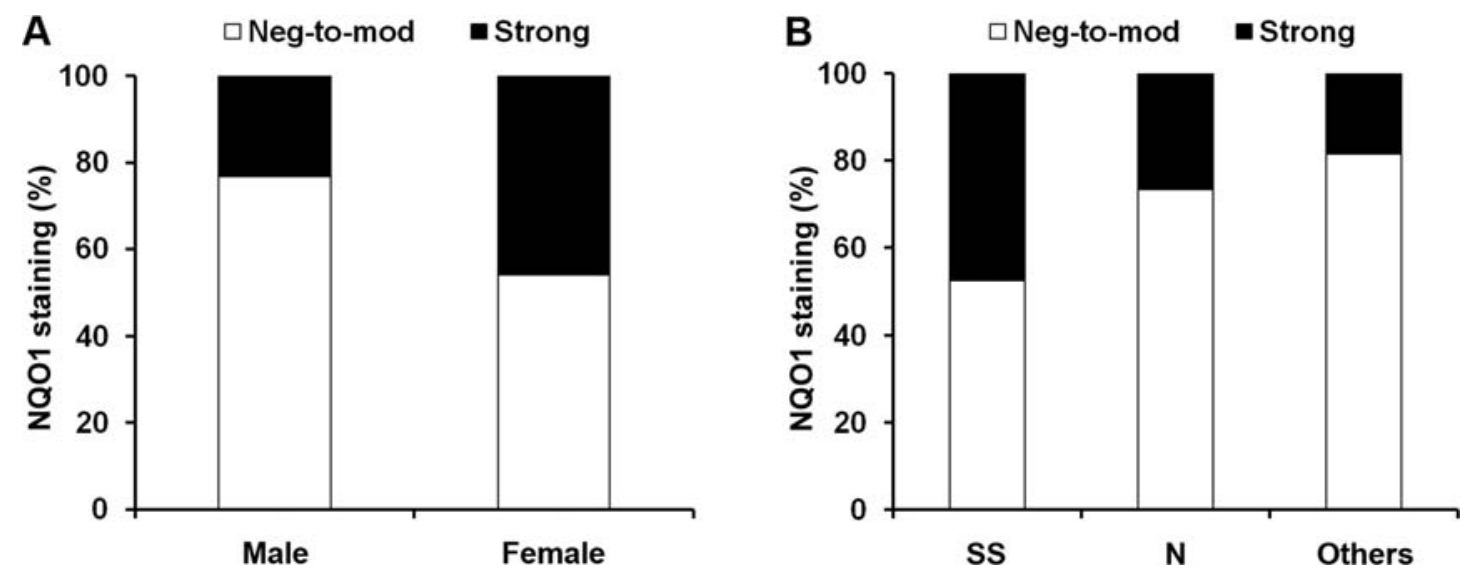

Figure 3. Correlation between NQO1 expression and clinicopathological parameters. (A) Female patients showed a significantly higher percentage of strong NQO1 expression than male patients ( $\mathrm{P}=0.022, \chi^{2}$ test). (B) NQO1 expression is significantly higher in superficial spreading melanomas than other subtypes $\left(\mathrm{P}=0.020, \chi^{2}\right.$ test $)$.

coverslips. Negative controls were performed by omitting NQO1 antibody during the primary antibody incubation.

Evaluation of immunostaining. The staining intensity and percentage of NQO1 positive cells were evaluated in a blinded manner by three independent observers (including one dermatopathologist) simultaneously, and a consensus score was reached for each core. Due to loss of biopsy cores, 56 cases of dysplastic nevi and 93 cases of primary melanoma could be evaluated for NQO1 staining. NQO1 staining intensity was scored 0-3 (0 negative; 1 weak; 2 moderate; 3 strong). The percentage of NQO1 positive cells was scored into 4 categories: 1 (0-25\%), 2 (26-50\%), 3 (51$75 \%)$, and $4(76-100 \%)$. In the cases with a discrepancy between duplicated cores, the higher score from the two tissue cores was taken as the final score. The multiplication of the intensity and percentage scores is used as the final staining score. The final score for NQO1 staining was defined as: negative (0), weak (1-4), moderate (6-9) and strong (12).

Statistical analysis of TMA. The SPSS version 11.5 software (SPSS, Chicago, IL) was used for the statistical analysis and all tests of statistical significance were two-sided. We used the $\chi^{2}$ test to compare the NQO1 staining intensity in different melanocytic lesions, as well as the correlation between NQO1 staining and the clinicopathological parameters of the melanoma patients, including age, gender, tumor thickness, ulceration, histological subtype and tumor location. The Kaplan-Meier survival curve and log-rank test were used to evaluate the correlations between NQO1 expression and patient survival. $\mathrm{P}<0.05$ was considered statistically significant.

\section{Results}

Clinicopathological features of TMAs. The clinicopathological features of the melanomas for this study are summarised in Table I. Ninety-three cases of primary melanoma (56 male and 37 female) were available for the evaluation of NQO1 staining. The median age of the patients was 57 years ranging from age 21 to 93 years. For melanoma staging, we used
Breslow thickness and AJCC stages as our criteria for NQO1 expression. In our study, 30 melanoma cases were $\leq 1 \mathrm{~mm}, 31$ were $1.01-2.0 \mathrm{~mm}, 14$ were $2.01-4.0 \mathrm{~mm}, 18$ were $>4 \mathrm{~mm}$ thick; 48 tumors were at AJCC stage I, 41 were at AJCC stage II, and four were at stage III. For the tumor subtype, there were 40 cases of superficial spreading melanoma, 15 cases of nodular melanoma, 38 cases of other subtypes, including lentigo mligna melanoma, acrolentigous melanoma, and desmoplastic melanoma. The majority of the melanomas (75 cases) were located in sun-protected sites (trunk, arm, leg and feet), while 18 cases located in sun-exposed sites (head and neck). Tumor ulceration was found in 13 patients.

NQO1 expression is increased in primary melanomas. We examined overall NQO1 staining in dysplastic nevi and primary melanomas by immunohistochemistry (Fig. 1). In primary melanoma, strong NQO1 staining was observed in 30 cases $(32.3 \%)$ while 63 cases $(67.7 \%)$ had negative-tomoderate NQO1 staining. In dysplastic nevi, 48 cases $(87 \%)$ had negative-to-moderate NQO1 expression, while only 8 cases $(14.3 \%)$ showed strong staining (Fig. 2). A significant difference in NQO1 staining was observed between dysplastic nevi and primary melanoma $\left(\mathrm{P}=0.015, \chi^{2}\right.$ test $)$.

Correlation between NQO1 expression and clinicopathological parameters or 5-year patient survival. To assess whether NQO1 expression correlates with clinicopathologicial parameters of the patients, we examined the expression of NQO1 in 93 primary melanomas at various stages of invasion. We analyzed the expression level of NQO1 in tumors with different thickness and AJCC stages, as tumor thickness and AJCC stages are well known prognostic markers for patients with primary melanomas. We did not find a significant correlation between NQO1 expression and tumor thickness or AJCC stages (Table I). Interestingly, strong NQO1 expression was detected in $54 \%$ of female patients compared to only $25 \%$ of male patients who had strong NQO1 expression $\left(\mathrm{P}=0.022, \chi^{2}\right.$ test; Fig. 3A). In addition, strong NQO1 expression was observed in $48 \%$ of superficial spreading melanomas compared with $27 \%$ of nodular melanomas and 
Table I. NQO1 expression and clinicopathological characteristics of 93 primary melanomas.

\begin{tabular}{|c|c|c|c|c|c|c|}
\hline & \multicolumn{4}{|c|}{ NQO1 staining } & \multirow[b]{2}{*}{ Total } & \multirow[b]{2}{*}{ P-value ${ }^{a}$} \\
\hline & Negative & Weak & Moderate & Strong & & \\
\hline \multicolumn{7}{|l|}{ Age } \\
\hline$\leq 57$ & $9(19 \%)$ & $10(22.3)$ & $8(17 \%)$ & $20(43 \%)$ & 47 & \\
\hline$>57$ & $8(17 \%)$ & $16(35 \%)$ & $12(26 \%)$ & $10(22 \%)$ & 46 & $\mathrm{P}>0.05$ \\
\hline \multicolumn{7}{|l|}{ Gender } \\
\hline Male & $14(25 \%)$ & $17(18 \%)$ & $12(32 \%)$ & $13(25 \%)$ & 56 & \\
\hline Female & $3(8 \%)$ & $9(22 \%)$ & $8(16 \%)$ & $17(54 \%)$ & 37 & $\mathrm{P}<0.05$ \\
\hline \multicolumn{7}{|c|}{ Tumor thickness (mm) } \\
\hline$\leq 1$ & $1(3 \%)$ & $8(27 \%)$ & $6(20 \%)$ & $15(50 \%)$ & 30 & \\
\hline $1.01-2$ & $5(16 \%)$ & $10(32 \%)$ & $8(26 \%)$ & $8(26 \%)$ & 31 & \\
\hline $2.01-4$ & $3(21 \%)$ & $4(29 \%)$ & $3(21 \%)$ & $4(29 \%)$ & 14 & \\
\hline$>4$ & $8(44 \%)$ & $4(22 \%)$ & $3(17 \%)$ & $3(17 \%)$ & 18 & $\mathrm{P}>0.05^{\mathrm{b}}$ \\
\hline \multicolumn{7}{|l|}{ Ulceration } \\
\hline Absent & $13(16 \%)$ & $24(30 \%)$ & $20(25 \%)$ & $23(29 \%)$ & 80 & \\
\hline Present & $4(31 \%)$ & $2(15 \%)$ & $0(0 \%)$ & $7(54 \%)$ & 13 & $\mathrm{P}>0.05$ \\
\hline \multicolumn{7}{|l|}{ Tumor subtype } \\
\hline SSM & $4(10 \%)$ & $7(18 \%)$ & $10(25 \%)$ & $19(48 \%)$ & 40 & \\
\hline NM & $4(27 \%)$ & $3(20 \%)$ & $4(27 \%)$ & $4(27 \%)$ & 15 & \\
\hline Other & $7(18 \%)$ & $17(45 \%)$ & $7(18 \%)$ & $7(18 \%)$ & 38 & $\mathrm{P}<0.05$ \\
\hline \multicolumn{7}{|l|}{ Site $^{\mathrm{d}}$} \\
\hline Sun-exposed & $4(22 \%)$ & $6(33 \%)$ & $3(17 \%)$ & $5(28 \%)$ & 18 & \\
\hline Sun-protected & $13(17 \%)$ & $20(27 \%)$ & $17(23 \%)$ & $25(33 \%)$ & 75 & $\mathrm{P}>0.05$ \\
\hline \multicolumn{7}{|l|}{ AJCC } \\
\hline I & $4(8 \%)$ & $13(27 \%)$ & $11(23 \%)$ & $20(42 \%)$ & 48 & \\
\hline II & $12(29 \%)$ & $12(29 \%)$ & $7(17 \%)$ & $10(24 \%)$ & 41 & \\
\hline III & $1(25 \%)$ & $1(25 \%)$ & $1(25 \%)$ & $1(25 \%)$ & 4 & $P>0.05$ \\
\hline
\end{tabular}

${ }^{\mathrm{a}} \chi^{2}$-test for negative-to-moderate versus strong NQO1 expression. ${ }^{\mathrm{b}}$ Tumors, $\leq 2 \mathrm{~mm}$ versus $>2 \mathrm{~mm}$. ${ }^{\mathrm{c} S S M}$, superficial spreading melanoma; NM, nodular melanoma; other includes desmoplastic melanoma, acrolentigous melanoma and lentigomeligna melanoma. ${ }^{\mathrm{d} S u n-p r o t e c t e d}$ sites: trunk, arm, leg and feet; sun-exposed sites: head and neck.

$18 \%$ of other tumor subtypes $\left(\mathrm{P}=0.020, \chi^{2}\right.$ test; Fig. $\left.3 \mathrm{~B}\right)$. No correlation was found between NQO1 expression and patient age, tumor site, or ulceration status of tumors (Table I). We then examined if NQO1 expression was associated with the survival of patients with primary melanoma by Kaplan-Meier survival analysis. Our results showed that NQO1 expression did not correlated with 5-year overall and disease-specific (Fig. 4) patient survival in primary melanoma ( $P>0.05$, longrank test).

Correlation between $\mathrm{NQO1}$ and $\mathrm{NF}-\kappa \mathrm{B}$ subunit $\mathrm{p} 50$ expression. Since our previous studies demonstrated that NF-кB p105/ p50 nuclear staining increased with melanoma progression,

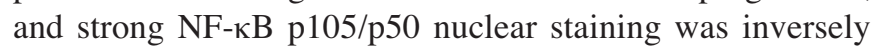
correlated with disease-specific 5-year survival of patients with tumor thickness $>2 \mathrm{~mm}$ (26), we examined the correlation of NQO1 and NF-кB p105/p50 nuclear expression in 92 cases of primary melanoma. As shown in Fig. 5, 46 cases $(61 \%)$ of strong NQO1 staining cases showed moderate to strong NF- $\mathrm{KB}$ staining; while only 5 cases $(31 \%)$ of negative to moderate NQO1 staining cases showed moderate to strong NF- $\mathrm{B}$ staining $\left(\mathrm{P}=0.032, \chi^{2}\right.$ test). Our data demonstrated that $\mathrm{NF}-\kappa \mathrm{B}$ nuclear staining is significantly increased with NQO1 expression (Fig. 5).

\section{Discussion}

An important phenomenon observed in a variety of human tumors at several sites is the increased expression of NQO1, suggesting that elevated levels of this enzyme may provide an effective biomarker for neoplasia (27). However, study on the role of NQO1 in melanoma is limited. The purpose of this study was to gain information on the role of NQO1 in melanoma progression. We used TMA technology and immunohistochemistry to investigate NQO1 expression in 93 primary melanoma and 56 dysplstic nevi biopsies. Results 
A

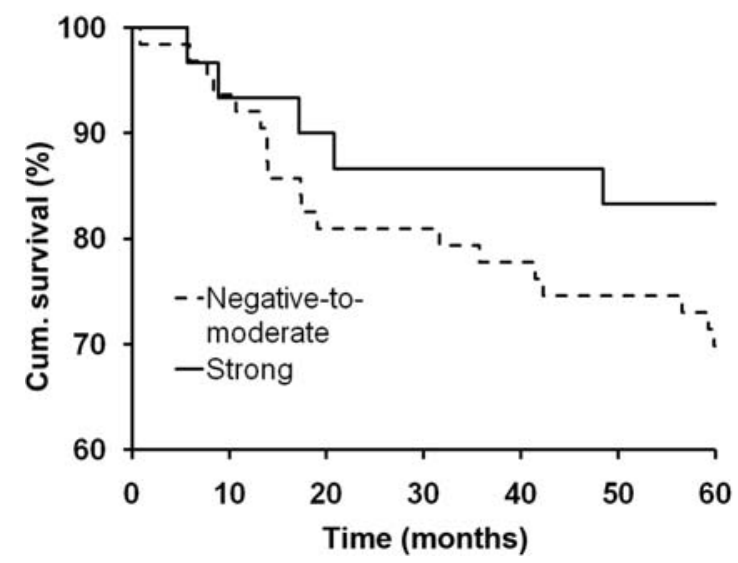

B

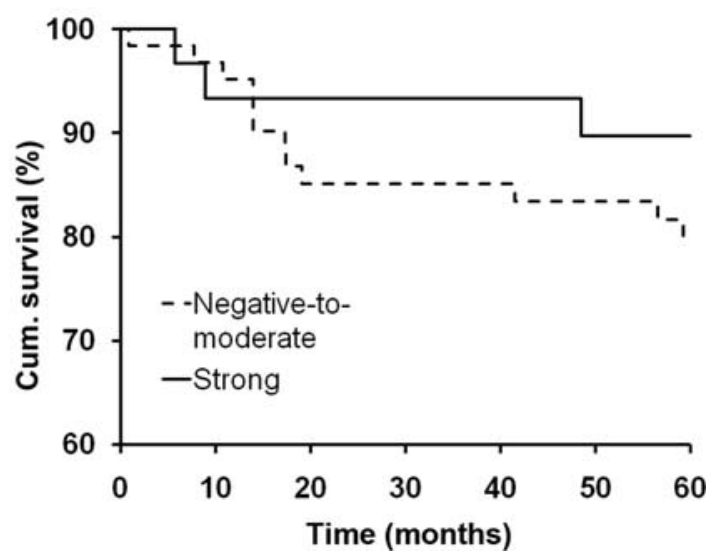

Figure 4. Correlation between NQO1 expression and 5-year survival of primary melanoma patients. There is no significant correlation of NQO1 expression with overall 5-year survival (A) and disease-specific 5-year survival (B) (P>0.05, long-rank test).

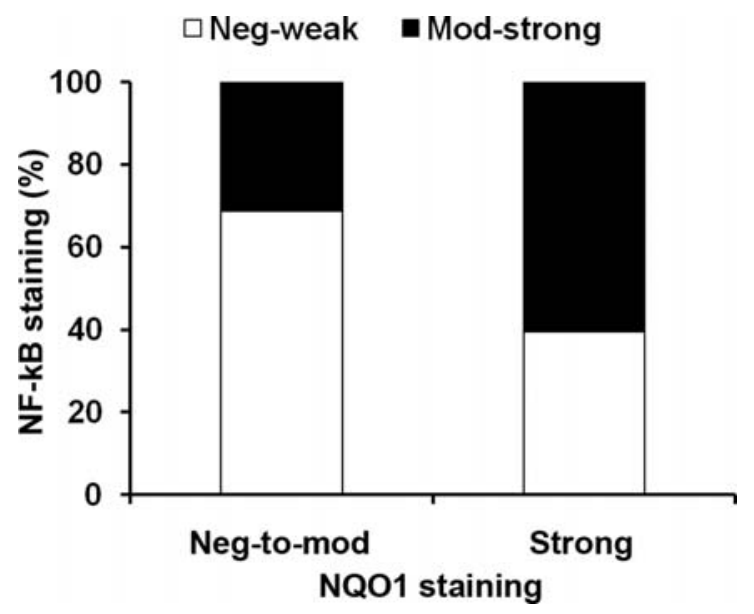

Figure 5. Correlation between NQO1 and NF-кB expression in 92 cases of primary melanoma. NF- $\mathrm{kB}$ expression was positively associated with NQO1 expression $\left(\mathrm{P}=0.032, \chi^{2}\right.$ test $)$.

from current study demonstrate that higher levels of NQO1 expression occur in primary melanoma tissue as has been reported for malignant tissue of the colon, breast, lung, pancreas, and liver when compared to normal tissue $(18,20,28,29)$. Our data imply the critical role of NQO1 in the initiation of melanomagenesis. NQO1 has long been viewed as a 'good' protein that protects humans from genotoxic damage. Additionally, it has been found that NQO1 is required in the stabilization of tumor suppressor $\mathrm{p} 53$, which supports the protective role of NQO1 against cancer (30). However, there is mounting evidence supporting a positive role for NQO1 in cancer progression. NQO1 is overexpressed in various tumors as mentioned above.

In another study, protein expression and enzyme activity of NQO1 was increased in colon and gastric carcinoma cell lines and in colorectal tumor samples compared to peripheral normal samples (22). These data indicate that NQO1 has potential dual roles in cancer. One possible explanation for high levels of NQO1 in tumors is that NQO1 protects not only normal cells from transforming into cancer cells, but also promotes the survival of cancer cells under a deleterious environment. Recent study in our laboratory demonstrates that NQO1 expression significantly induces cell cycle progression by upregulating the expression of cyclin A2, B1 and D1, leading to the proliferation of melanoma cells, which may account for the overexpression of NQO1 in primary melanoma (unpublished data). In addition, NQO1 overexpression is thought to be accompanied by an increase in other antioxidant enzymes, such as HMOX-1 and GST, providing tumors with increased protection against cytotoxic agents allowing for rapid cancer progression (31). However, we did not find any significant correlation of NQO1 expression with tumor thickness, AJCC stages (Table I), or 5-year overall and disease-specific patient survival in primary melanomas (Fig. 4).

Interestingly, there was a higher percentage of strong NQO1 expression in female patients than male patients (Fig. 3A). This finding is consistent with the result of a study conducted on rats. In Sprague Dawley rats, gender dimorphic expression of NQO1 was observed with female mRNA, protein, and activity levels being significantly higher than in males (32). As shown by many studies, male patients may have thicker tumors at diagnosis than female patients (33-35). However, in our study, when we compared the number of tumors $\leq 2.0 \mathrm{~mm}$ or $>2.0 \mathrm{~mm}$, there was no difference between male and female groups $\left(\mathrm{P}=0.75, \chi^{2}\right.$ test $)$. We also found higher expression of NQO1 in superficial spreading melanomas than nodular or other types melanomas $\left(\mathrm{P}=0.020, \chi^{2}\right.$ test $)$. A recent microarray study of primary and metastatic melanoma indicated that superficial spreading and nodular melanoma had different gene expression patterns (36). Different NQO1 expression level is probably due to the genetic difference among the subtypes. The mechanisms of higher NQO1 expression in female patients and superficial spreading melanomas, and their clinical significance remain to be determined.

The factors underlying the increased levels of NQO1 in primary melanoma tissue need to be established. Expression of NQO1 is known to be induced in response to a variety of xenobiotics including polycyclic aromatic hydrocarbons, planar aromatic compounds, antioxidants, oxidants, and tumor promoters (37). As a key enzyme involved in defense against 
reactive forms of oxygen, expression of NQO1 is thought to be transcriptionally controlled. Several cis-elements have been identified in the NQO1 gene promoter, including an antioxidant response element (ARE), a basal element, and AP2 element. Nuclear transcription factors Nrf2 and c-Jun bind to the ARE and activate gene expression. A NF- $\mathrm{B}$ response element may also be involved in NQO1 induction (24).

Recent studies suggested that NF-KB is the major transcription factor that has been shown to play a critical role in the modulation of $N Q O 1$. Several studies also showed that induction of $N Q O 1$ gene by hypoxia and mitomycin $\mathrm{C}$ in human colon adenocarcinoma HT29 and human hepatoma HepG2 cells is mediated through a mechanism involving the $\mathrm{NF}-\kappa \mathrm{B}$ signaling pathway $(23,24)$. NF- $\kappa \mathrm{B}$ has been shown to play an important role in melanoma cell proliferation, resistance to apoptosis, invasion, and metastasis (25). In addition, our previous studies have demonstrated that NF-кB p105/p50 nuclear staining increased with melanoma progression (26). Based on these findings, we investigated the correlation between NQO1 and NF- $\mathrm{KB}$ expression. Using the same tissue microarray and same protocol for immunohistochemistry staining, our analysis revealed a significant correlation between NQO1 and NF-кB expression (Fig. 5). The data indicated that $\mathrm{NF}-\kappa \mathrm{B}$ signaling pathway may be involved in the induction of $N Q O 1$ gene expression. NF- $\mathrm{KB}$ is a pivotal transcription factor involved in a wide range of physiological functions, such as B-cell proliferation, cell cycle control, apoptosis, and carcinogenesis $(38,39)$. Many stimuli activate $\mathrm{NF}-\kappa \mathrm{B}$, including cytokines, activators of protein kinase $C$, viruses, and oxidants $(40,41)$. Upon activation, $\mathrm{NF}-\kappa \mathrm{B}$ regulates the expression of many genes involved in cell proliferation, tumor invasion, immune and inflammatory responses (41). NQO1 is probably one of $N F-\kappa B$ regulated genes involved in cell proliferation but not in tumor invasion.

Collectively, our data indicate that NQO1 expression is significantly increased in primary melanoma compared to dysplastic nevi, but not correlated to tumor thickness or patient survival, suggesting that NQO1 may play an important role in melanoma initiation.

\section{Acknowledgements}

This work was supported by Canadian Institutes of Health Research (MOP-84559 and MOP-93810) and Canadian Dermatology Foundation.

\section{References}

1. Arenberger P, Arenbergerova M and Vedral T: Malignant melanoma: standards and innovation in diagnostics and therapy. Cas Lek Cesk 148: 151-157, 2009.

2. Arora A and Attwood J: Common skin cancers and their precursors. Surg Clin North Am 89: 703-712, 2009.

3. Doben AR and MacGillivray DC: Current concepts in cutaneous melanoma: malignant melanoma. Surg Clin North Am 89: 713-725, 2009.

4. Houghton AN and Polsky D: Focus on melanoma. Cancer Cell 2: 275-278, 2002.

5. Balch CM, Buzaid AC, Soong SJ, et al: Final version of the American Joint Committee on Cancer staging system for cutaneous melanoma. J Clin Oncol 19: 3635-3648, 2001.

6. Tsao H: Management of cutaneous melanoma. New Engl J Med 351: 2461, 2004

7. Rigel DS: The effect of sunscreen on melanoma risk. Dermatol Clin 20: 601-606, 2002.
8. Manola J, Atkins M, Ibrahim J and Kirkwood J: Prognostic factors in metastatic melanoma: a pooled analysis of Eastern Cooperative Oncology Group trials. J Clin Oncol 18: 3782-3793, 2000.

9. Powis G, Svingen BA and Appel P: Quinone-stimulated superoxide formation by subcellular fractions, isolated hepatocytes, and other cells. Mol Pharmacol 20: 387-394, 1981

10. Lind C, Cadenas E, Hochstein P and Ernster L: DT-diaphorase: purification, properties, and function. Methods Enzymol 186: 287-301, 1990.

11. Chesis PL, Levin DE, Smith MT, Ernster L and Ames BN: Mutagenicity of quinones: pathways of metabolic activation and detoxification. Proc Natl Acad Sci USA 81: 1696-1700, 1984.

12. Lind C, Hojeberg B, Seidegard J, DePierre JW and Ernster L: Induction of hepatic cytosolic DT diaphorase in rats treated with trans-stilbene oxide. FEBS Lett 116: 289-292, 1980.

13. Thor H, Smith MT, Hartzell P, Bellomo G, Jewell SA and Orrenius S: The metabolism of menadione (2-methyl-1,4naphthoquinone) by isolated hepatocytes. A study of the implications of oxidative stress in intact cells. J Biol Chem 257: 12419-12425, 1982.

14 Siegel D and Ross D: Immunodetection of NAD $(\mathrm{P}) \mathrm{H}$ :quinone oxidoreductase 1 (NQO1) in human tissues. Free Radic Biol Med 29: 246-253, 2000.

15. Ross D, Siegel D, Beall H, Prakash AS, Mulcahy RT and Gibson NW: DT-diaphorase in activation and detoxification of quinones. Bioreductive activation of mitomycin C. Cancer Metastasis Rev 12: 83-101, 1993.

16. Workman P: Enzyme-directed bioreductive drug development revisited: a commentary on recent progress and future prospects with emphasis on quinone anticancer agents and quinone metabolizing enzymes, particularly DT-diaphorase. Oncol Res 6: 461-475, 1994.

17. De Flora S, Bennicelli C, D'Agostini F, Izzotti A and Camoirano A: Cytosolic activation of aromatic and heterocyclic amines. Inhibition by dicoumarol and enhancement in viral hepatitis B. Environ Health Perspect 102 (Suppl 6): 69-74, 1994.

18. Malkinson AM, Siegel D, Forrest GL, et al: Elevated DTdiaphorase activity and messenger RNA content in human nonsmall cell lung carcinoma: relationship to the response of lung tumor xenografts to mitomycin $\mathrm{Cl}$. Cancer Res 52: 4752-4757, 1992.

19. Mekhail-Ishak K, Hudson N, Tsao MS and Batist G: Implications for therapy of drug-metabolizing enzymes in human colon cancer. Cancer Res 49: 4866-4869, 1989.

20. Schlager JJ and Powis G: Cytosolic NAD(P)H:(quinoneacceptor)oxidoreductase in human normal and tumor tissue: effects of cigarette smoking and alcohol. Int J Cancer 45: 403-409, 1990.

21. Smitskamp-Wilms E, Giaccone G, Pinedo HM, van der Laan BF and Peters GJ: DT-diaphorase activity in normal and neoplastic human tissues; an indicator for sensitivity to bioreductive agents? Br J Cancer 72: 917-921, 1995.

22. Mikami K, Naito M, Ishiguro T, et al: Immunological quantitation of DT-diaphorase in carcinoma cell lines and clinical colon cancers: advanced tumors express greater levels of DT-diaphorase. Jpn J Cancer Res 89: 910-915, 1998.

23. Yao KS and O'Dwyer PJ: Involvement of NF-kappa B in the induction of $\mathrm{NAD}(\mathrm{P}) \mathrm{H}$ :quinone oxidoreductase (DT-diaphorase) by hypoxia, oltipraz and mitomycin C. Biochem Pharmacol 49: 275-282, 1995.

24. Yao KS, Hageboutros A, Ford P and O'Dwyer PJ: Involvement of activator protein-1 and nuclear factor-kappaB transcription factors in the control of the DT-diaphorase expression induced by mitomycin C treatment. Mol Pharmacol 51: 422-430, 1997.

25. Amiri KI and Richmond A: Role of nuclear factor-kappa B in melanoma. Cancer Metastasis Rev 24: 301-313, 2005.

26. Gao K, Dai DL, Martinka M and Li G: Prognostic significance of nuclear factor-kappaB p105/p50 in human melanoma and its role in cell migration. Cancer Res 66: 8382-8388, 2006.

27. Lyn-Cook BD, Yan-Sanders Y, Moore S, Taylor S, Word B and Hammons GJ: Increased levels of NAD(P)H: quinone oxidoreductase 1 (NQO1) in pancreatic tissues from smokers and pancreatic adenocarcinomas: A potential biomarker of early damage in the pancreas. Cell Biol Toxicol 22: 73-80, 2006.

28. Koudstaal J, Makkink B and Overdiep SH: Enzyme histochemical pattern in human tumours. II. Oxidoreductases in carcinoma of the colon and the breast. Eur J Cancer 11: 111-115, 1975. 
29. Schor NA and Cornelisse CJ: Biochemical and quantitative histochemical study of reduced pyridine nucleotide dehydrogenation by human colonic carcinomas. Cancer Res 43: 4850-4855, 1983.

30. Asher G, Lotem J, Cohen B, Sachs L and Shaul Y: Regulation of p53 stability and p53-dependent apoptosis by NADH quinone oxidoreductase 1. Proc Natl Acad Sci USA 98: 1188$1193,2001$.

31. Lau A, Villeneuve NF, Sun Z, Wong PK and Zhang DD: Dual roles of Nrf2 in cancer. Pharmacol Res 58: 262-270, 2008

32. Augustine LM, Fisher CD, Lickteig AJ, Aleksunes LM, Slitt AL and Cherrington NJ: Gender divergent expression of Nqo1 in Sprague Dawley and August Copenhagen x Irish rats. J Biochem Mol Toxicol 22: 93-100, 2008

33. Melia J, Cooper EJ, Frost T, et al: Cancer Research Campaign health education programme to promote the early detection of cutaneous malignant melanoma. II. Characteristics and incidence of melanoma. Br J Dermatol 132: 414-421, 1995.

34. Hersey P, Sillar RW, Howe CG, et al: Factors related to the presentation of patients with thick primary melanomas. Med J Aust 154: 583-587, 1991.

35. Osborne JE and Hutchinson PE: Clinical correlates of Breslow thickness of malignant melanoma. Br J Dermatol 144: 476-483, 2001.
36. Jaeger J, Koczan D, Thiesen HJ, et al: Gene expression signatures for tumor progression, tumor subtype, and tumor thickness in laser-microdissected melanoma tissues. Clin Cancer Res 13: 806-815, 2007.

37. Dhakshinamoorthy S, Long DJ II and Jaiswal AK: Antioxidant regulation of genes encoding enzymes that detoxify xenobiotics and carcinogens. Curr Top Cell Regul 36: 201-216, 2000.

38. Shen G, Jeong WS, Hu R and Kong AN: Regulation of Nrf2, NF-kappaB, and AP-1 signaling pathways by chemopreventive agents. Antioxid Redox Signal 7: 1648-1663, 2005.

39. Korashy HM and El-Kadi AO: NF-kappaB and AP-1 are key signaling pathways in the modulation of $\mathrm{NAD}(\mathrm{P}) \mathrm{H}$ :quinone oxidoreductase 1 gene by mercury, lead, and copper. J Biochem Mol Toxicol 22: 274-283, 2008.

40. Siebenlist U, Franzoso G and Brown K: Structure, regulation and function of NF-kappa B. Annu Rev Cell Biol 10: 405-455, 1994.

41. Baeuerle PA and Baltimore D: NF-kappa B: ten years after. Cell 87: 13-20, 1996. 